\title{
AN APPLICATION OF THE THEORY OF SCALE OF BANACH SPACES
}

\author{
ŁUKASZ DAWIDOWSKI
}

\begin{abstract}
The abstract Cauchy problem on scales of Banach space was considered by many authors. The goal of this paper is to show that the choice of the space on scale is significant. We prove a theorem that the selection of the spaces in which the Cauchy problem $u_{t}-\Delta u=u|u|^{s}$ with initialboundary conditions is considered has an influence on the selection of index $s$. For the Cauchy problem connected with the heat equation we will study how the change of the base space influents the regularity of the solutions.
\end{abstract}

\section{Introduction}

Fractional powers of a positive sectorial operator $A$ defined on Banach space $X$ and scale of Banach space generated by the powers of operator $A$ were considered in the papers [7], [2], [3]. We would like to prove that choice of the base space affect the selection of assumptions.

Let us consider the abstract Cauchy problem

$$
\left\{\begin{array}{l}
u_{t}+A u=F(u), \quad t>0 \\
u(0)=u_{0}
\end{array}\right.
$$

where $A$ is sectorial in a Banach space $X$. Without loss of generality (adding - if necessary - a term $c u$ to both sides of (1.1p), we may assume that $A$ is

Received: 13.04.2015. Revised: 9.05.2015.

(2010) Mathematics Subject Classification: Primary 46B70; Secondary 35K10.

Key words and phrases: scale of Banach spaces, parabolic equations. 
sectorial and positive; $\operatorname{Re} \sigma(A)>0$. We will search for local $X^{z}$-solution of the problem 1.1 in the sense precised below.

Definition 1.1. Let $X$ be a Banach space, $z \in[0,1)$ and $u_{0}$ be an element of $X^{z}$. If, for some real $\tau>0$, a function $u \in C\left([0, \tau), X^{z}\right)$ satisfies

$$
\begin{aligned}
& u(0)=u_{0}, \\
& u \in C^{1}((0, \tau), X), \\
& u(t) \in D(A) \text { for all } t \in(0, \tau), \\
& u \text { solves the first equation in (1.1) in } X \text { for all } t \in(0, \tau),
\end{aligned}
$$

then $u$ is called a local $X^{z}$-solution of the problem (1.1).

The following theorem gives conditions for the existence of local $X^{z}$ solution.

Theorem 1.2. Let $X$ be a Banach space, $A: D(A) \rightarrow X$ a sectorial operator in $X$ with $\operatorname{Re} \sigma(A)>0$. Let $F: X^{z} \rightarrow X$ be Lipschitz continuous on bounded subsets of $X^{z}$ for some $z \in[0,1)$. Then, for each $u_{0} \in X^{z}$, there exists a unique $X^{z}$-solution $u=u\left(t, u_{0}\right)$ of (1.1) defined on its maximal interval of existence $\left[0, \tau_{u_{0}}\right)$, which means that either $\tau_{u_{0}}=+\infty$ or

$$
\text { if } \tau_{u_{0}}<+\infty \text { then } \limsup _{t \rightarrow \tau_{u_{0}}^{-}}\left\|u\left(t, u_{0}\right)\right\|_{X^{z}}=+\infty \text {. }
$$

We can find the proof of this Theorem in [6, Section 3.3].

\section{Sectoriality of operator}

Assume that $(X,\|\cdot\|)$ is a Banach space, and let $A: D(A) \subseteq X \rightarrow X$ be a sectorial operator in $X$. We consider a scale of the Banach spaces $\left(X^{\alpha}\right)_{\alpha \in \mathbb{R}}$, generated by operator $A$ in the space $X$ (for details see [7] or [3, Chapter 1]). Then, for $\alpha \in \mathbb{R}$, we can consider operators $\left.A\right|_{X^{\alpha}}: X^{\alpha+1} \subseteq X^{\alpha} \rightarrow X^{\alpha}$ given by

(i) if $\alpha=0$, then an operator $\left.A\right|_{X}: X^{1} \subseteq X \rightarrow X$ coincides with $A: D(A) \subseteq$ $X \rightarrow X$,

(ii) if $\alpha>0$, then $X^{\alpha}=D\left(A^{\alpha}\right)$ is a dense subset of $X$ and $X^{\alpha+1}$ is densely contained in $X^{1}$, so we can consider an operator $\left.A\right|_{X^{\alpha}}: X^{\alpha+1} \subseteq X^{\alpha} \rightarrow$ $X^{\alpha}$ as a restriction of $A: D(A) \subseteq X \rightarrow X$ to the set $X^{\alpha+1}$, i.e.

$$
\left.A\right|_{X^{\alpha}} x=A x, \quad x \in X^{\alpha+1},
$$


(iii) if $\alpha<0$, then the space $X^{\alpha}$ is a completion of the space $X$ in the norm $\|\cdot\|_{X^{\alpha}}$, so we can consider an operator $\left.A\right|_{X^{\alpha}}: X^{\alpha+1} \subseteq X^{\alpha} \rightarrow X^{\alpha}$ as a realization (extension) of $A: D(A) \subseteq X \rightarrow X$, i.e.

$$
\left.A\right|_{X^{\alpha}} x=A x, \quad x \in X^{\alpha+1} \cap X .
$$

We present lemma which says that when an operator $A: D(A) \subseteq X \rightarrow X$ is sectorial, then all the operators $\left.A\right|_{X^{\alpha}}: X^{\alpha+1} \subseteq X^{\alpha} \rightarrow X^{\alpha}$ for $\alpha \in \mathbb{R}$ are also sectorial.

Lemma 2.1. When $\left.A\right|_{X}: X^{1} \subseteq X \rightarrow X$ is a sectorial operator with $\operatorname{Re} \sigma\left(\left.A\right|_{X}\right)>0$, then all operators $\left.A\right|_{X^{\alpha}}: X^{\alpha+1} \subseteq X^{\alpha} \rightarrow X^{\alpha}$ for $\alpha \in \mathbb{R}$ are sectorial.

The proof of this Lemma can be found in [3, pp. 80-81].

\section{Applications}

The operator $-\Delta$ defined in the space $L^{p}(\Omega)$ with $D(-\Delta)=W^{2, p}(\Omega) \cap$ $W_{0}^{1, p}(\Omega)$, where $\Omega \subseteq \mathbb{R}^{n}$ is a bounded domain and $p \in[1, \infty)$, is sectorial (see [8, Theorems 2.12 and 2.13, pp. 77,79]). Then using Theorem 1.2 to get a solution of problem

$$
\left\{\begin{array}{l}
u_{t}-\Delta u=f(u), \\
\left.u\right|_{\partial \Omega}=0, \\
u(0, x)=u_{0} \in X^{z},
\end{array}\right.
$$

we have to show that the function $f: X^{z} \rightarrow X$ satisfies Lipschitz condition on bounded subsets of $X^{z}(z \in[0,1))$. It is often easier to verify this condition in a bigger space on the scale.

Let us take $X=L^{p}(\Omega)$ where $\Omega \subseteq \mathbb{R}^{n}$ is a bounded domain with $\partial \Omega \in C^{2}$ and $p \in[1, \infty)$. Then the Banach scale generated by the space $X$ and operator $-\Delta$ is in the form $X^{\gamma}=W_{B}^{2 \gamma, p}(\Omega)$ for $\gamma \in \mathbb{R}$ (see [2, 3]), where $W_{B}^{2 \gamma, p}(\Omega)$ denotes the fractional Sobolev space with Dirichlet boundary conditions (for more details see [8, Section 16.6.2]). If we choose the base space as $Y=X^{-\gamma}=$ $W_{B}^{-2 \gamma, p}(\Omega)$ for some $\gamma \in\left[0, \frac{1}{2}\right]$, then we will have to prove that the function $f: Y^{z} \rightarrow Y$, so $f: X^{-\gamma+z} \rightarrow X^{-\gamma}$ is Lipschitz continuous on bounded subsets of $X^{-\gamma+z}(z \in[0,1))$. 
Further, we will consider exemplary nonlinearity $f$ given by:

$$
f(u)=u|u|^{s},
$$

where $s \in \mathbb{R}^{+}$. We would like to find the largest possible value of $s$ such that $f$ is Lipschitz continuous on bounded subsets of $Y^{z}=X^{-\gamma+z}$ with certain $z \in[0,1)$.

Lemma 3.1. Let $\Omega \subseteq \mathbb{R}^{n}$ be a bounded domain with $\partial \Omega \in C^{2}$ and $p \in$ $[1, \infty)$. Let $z \in[0,1)$. Then function $f: W_{B}^{-2 \gamma+2 z, p}(\Omega) \rightarrow W^{-2 \gamma, p}(\Omega), f(u)=$ $u|u|^{s}$ with $s \in \mathbb{R}^{+}$is Lipschitz continuous on bounded subsets of $W_{B}^{-2 \gamma+2 z, p}(\Omega)$ if $s \leq s_{0}=\frac{2 z p}{n+2 \gamma p-2 z p}$ (or for each $s \in \mathbb{R}^{+}$if $n+2 \gamma p-2 z p \leq 0$ ).

Proof. We have (using mean value theorem) the following estimate:

$$
\begin{aligned}
|f(u)-f(v)| & =\left.|u| u\right|^{s}-v|v|^{s}|\leq| u|||u|^{s}-|v|^{s}|+| u-\left.v|| v\right|^{s} \\
& \leq|u| s|u-v|\left(|u|^{s-1}+|v|^{s-1}\right)+|u-v||v|^{s} \\
& =|u-v|\left(s|u|^{s}+s|u||v|^{s-1}+|v|^{s}\right) \\
& \leq c_{s}|u-v|\left(|u|^{s}+|v|^{s}\right) .
\end{aligned}
$$

Our aim is to estimate the difference $\|f(u)-f(v)\|_{W^{-2 \gamma, p}(\Omega)}$. For that purpose, going through the dual spaces, we first observe that $L^{\frac{n p}{n+2 \gamma p}}(\Omega) \subseteq W^{-2 \gamma, p}(\Omega)$. To prove this fact, we will show the corresponding embedding for dual spaces $W^{2 \gamma, p^{\prime}}(\Omega) \subseteq L^{r^{\prime}}(\Omega)$ where $p^{\prime}$ is Hölder conjugate exponent to $p\left(\frac{1}{p}+\frac{1}{p^{\prime}}=1\right)$ and $r^{\prime}$ to $\frac{n p}{n+2 \gamma p}$. From the Sobolev's theorem (see Theorem 6 (General Sobolev inequalities) in [4], page 270) we know that this embedding holds when $\frac{1}{r^{\prime}} \geq$ $\frac{1}{p^{\prime}}-\frac{2 \gamma}{n}$ and $1<\frac{n}{2 \gamma p^{\prime}}$. If we take $r^{\prime}=\frac{-n p}{2 \gamma p-n p+n}$, we will have

$$
\begin{aligned}
\frac{1}{r^{\prime}}=\frac{2 \gamma p-n p+n}{-n p} & =\frac{2 \gamma p}{-n p}+\frac{n-n p}{-n p} \\
& =\frac{p-1}{p}-\frac{2 \gamma}{n}=\frac{1}{p^{\prime}}-\frac{2 \gamma}{n},
\end{aligned}
$$

so that the embedding $W^{2 \gamma, p^{\prime}}(\Omega) \subseteq L^{r^{\prime}}(\Omega)$ holds.

Let us fix a bounded set $B \subseteq W_{B}^{-2 \gamma+2 z, p}(\Omega)$ and let $u, v \in B$. Let us choose $R=\frac{2 \gamma p+n}{n+2 \gamma p-2 z p}, Q=\frac{2 \gamma p+n}{2 z p}$ so that $\frac{1}{R}+\frac{1}{Q}=1$. Then using Hölder 
inequality, with exponents $R$ and $Q$, we obtain:

$$
\begin{aligned}
\|f(u)-f(v)\|_{W^{-2 \gamma, p}(\Omega)} & \leq c_{B}\|f(u)-f(v)\|_{L^{\frac{n p}{n+2 \gamma p}}(\Omega)} \\
& =c_{B}\left\|u|u|^{s}-v|v|^{s}\right\|_{L^{\frac{n p}{n+2 \gamma p}}(\Omega)} \\
& =c_{B}\left(\left.\int_{\Omega}|u| u\right|^{s}-\left.v|v|^{s}\right|^{\frac{n p}{n+2 \gamma p}} d x\right)^{\frac{n+2 \gamma p}{n p}} \\
\leq & c_{B}\left(\int_{\Omega}|| u-v\left|c_{s}\left(|u|^{s}+|v|^{s}\right)\right|^{\frac{n p}{n+2 \gamma p}} d x\right)^{\frac{n+2 \gamma p}{n p}} \\
\leq & c_{B} c_{s}\left(\int_{\Omega}|u-v|^{\frac{n p}{n+2 \gamma p} \cdot R} d x\right)^{\frac{1}{R} \frac{n+2 \gamma p}{n p}} \times \\
& \times\left(\int_{\Omega}(2 \max (|u|,|v|))^{s \cdot \frac{n p}{n+2 \gamma p} \cdot Q} d x\right)^{\frac{1}{Q} \frac{n+2 \gamma p}{n p}} .
\end{aligned}
$$

To assure that the second integral is finite we have to find the largest $s$ such that $W^{-2 \gamma+2 z, p}(\Omega) \subseteq L^{s \frac{n p}{2 \gamma p+n} Q}(\Omega)$. For that purpose, if we take $-2 \gamma+2 z \geq \frac{n}{p}$, we verify that $W^{-2 \gamma+2 z, p}(\Omega) \subseteq L^{\infty}(\Omega) \subseteq L^{s \frac{n p}{2 \gamma p+n} Q}(\Omega)$ for each $s \geq 0$, since $0 \leq-2 \gamma+2 z-\frac{n}{p}$ and $\Omega$ is a bounded set. On the second hand if we take $-2 \gamma+2 z<\frac{n}{p}, s_{0}=\frac{2 z p}{n+2 \gamma p-2 z p}$ and it is enough to check for $s \leq s_{0}$ the condition

$$
\begin{aligned}
-\frac{n}{s \frac{n p}{2 \gamma p+n} Q} & \leq-\frac{n}{\frac{2 z p}{n+2 \gamma p-2 z p} \frac{n p}{2 \gamma p+n} \frac{2 \gamma p+n}{2 z p}} \\
& =-\frac{n+2 \gamma p-2 z p}{p} \\
& =2 \gamma-2 z-\frac{n}{p} .
\end{aligned}
$$

Since $u, v$ are elements of the set $B$ which is bounded in $W^{-2 \gamma+2 z, p}(\Omega)$, hence bounded in $L^{s \frac{n p}{2 \gamma p+n} Q}(\Omega)$ for $s \leq s_{0}$, we observe that the second integral $\int_{\Omega}(2 \max (|u|,|v|))^{s \cdot \frac{n p}{n+2 \gamma p} \cdot Q} d x$ is finite and bounded by some constant. Therefore our estimation has the form

$$
\begin{aligned}
\|f(u)-f(v)\|_{W^{-2 \gamma, p}(\Omega)} & \leq c_{s, B}\left(\int_{\Omega}|u-v|^{\frac{n p}{n+2 \gamma p} \cdot R} d x\right)^{\frac{1}{R} \frac{n+2 \gamma p}{n p}} \\
& =c_{s, B}\|u-v\|_{L^{\frac{n p}{n+2 \gamma p} R}(\Omega)} .
\end{aligned}
$$


In the end we notice that $W^{-2 \gamma+2 z, p}(\Omega) \subseteq L^{\frac{n p}{n+2 \gamma p} R}(\Omega)$, since

$$
\begin{aligned}
-\frac{n}{\frac{n p}{n+2 \gamma p} R} & =-\frac{n}{\frac{n p}{n+2 \gamma p} \frac{2 \gamma p+n}{n+2 \gamma p-2 z p}} \\
& =-\frac{n+2 \gamma p-2 z p}{p} \\
& =-2 \gamma+2 z-\frac{n}{p} .
\end{aligned}
$$

This finishes the proof of Lipschitz continuity of $f$ on bounded subsets of $W_{B}^{-2 \gamma+2 z, p}(\Omega)$ because

$$
\begin{aligned}
\|f(u)-f(v)\|_{W^{-2 \gamma, p}(\Omega)} & \leq c_{s, B}\|u-v\|_{L^{\frac{n p}{n+2 \gamma p} R}(\Omega)} \\
& \leq \tilde{c}_{s, B}\|u-v\|_{W^{-2 \gamma+2 z, p}(\Omega)} .
\end{aligned}
$$

REMARK 3.2. Let us consider a critical exponent $z=1$. Then we can prove (in a very similar way) that nonlinearity $f: W_{B}^{-2 \gamma+2, p}(\Omega) \rightarrow W^{-2 \gamma, p}(\Omega)$, $f(u)=u|u|^{s}$ is Lipschitz continuous on bounded subsets of $W_{B}^{-2 \gamma+2, p}(\Omega)$ if $s \leq s_{0}=\frac{2 p}{n+2 \gamma p-2 p}$ (or for each $s \in \mathbb{R}^{+}$if $n+2 \gamma p-2 p \leq 0$ ).

As a consequence of previous lemma, following theorem holds:

TheOREM 3.3. Let $\Omega \subseteq \mathbb{R}^{n}$ be a bounded domain with $\partial \Omega \in C^{2}, p \in$ $[1, \infty)$ and $X^{\gamma}=W_{B}^{2 \gamma, p}(\Omega)$ be the Banach scale. Let $z \in[0,1)$. Let us consider the problem 3.1 in the space $Y=X^{-\gamma}=W_{B}^{-2 \gamma, p}(\Omega)$ with function $f: W_{B}^{-2 \gamma+2 z, p}(\Omega) \rightarrow W^{-2 \gamma, p}(\Omega), f(u)=u|u|^{s}$ with $s \in \mathbb{R}^{+}$. Then the problem 3.1 has a local $Y^{z}$-solution if $s \leq s_{0}=\frac{2 z p}{n+2 \gamma p-2 z p}$ (or for each $s \in \mathbb{R}^{+}$if $n+2 \gamma p-2 z p \leq 0)$.

REMARK 3.4. These results are generalization of examples given in the monograph [3] (Example 8.1.5 and Example 8.1.7). If we take $\gamma=0$, we see that the function $f: W_{B}^{2, p}(\Omega) \rightarrow L^{p}(\Omega), f(u)=u|u|^{s}$ is Lipschitz continuous on bounded subsets when $s \leq \frac{2 p}{n-2 p}$. But when we take $\gamma=\frac{1}{2}$, we will obtain that $f: W_{B}^{1, p}(\Omega) \rightarrow W^{-1, p}(\Omega), f(u)=u|u|^{s}$ will be Lipschitz continuous on bounded subsets if $s \leq \frac{2 p}{n-p}$.

We can remark, that when $\gamma \in\left[0, \frac{1}{2}\right]$ is bigger, so the space $W^{-2 \gamma, p}(\Omega)$ is bigger, then we will be able to take smaller index $s$ in nonlinearity $f$. 


\section{More regular solutions}

In this section we show that selection of base space can affect the regularity of solution of considered problem. In the following examples expected solution has to be more regular.

EXAMPLE 4.1. Let $\Omega \subseteq \mathbb{R}^{n}$ be a bounded domain with $\partial \Omega \in C^{2}$ and let us consider the Cauchy problem

$$
\left\{\begin{array}{l}
u_{t}-\Delta u=0 \\
\left.u\right|_{\partial \Omega}=0
\end{array}\right.
$$

An abstract operator $A: D(A) \subseteq X \rightarrow X$ in the base space $X$ will be defined by the differential operator $-\Delta$ subjected to suitable boundary conditions. Usually we take set $X=W_{0}^{1, p}(\Omega)$ and the domain $D(A)=W_{B}^{3, p}(\Omega)$. However we can select the base space in another way, taking

$$
X^{1}=D(A)=W_{B}^{3, p}(\Omega)
$$

and defining the base space as

$$
X^{\alpha}=\left[X, X^{1}\right]_{\alpha}, \quad \alpha \in(0,1),
$$

where $[\cdot, \cdot]_{\alpha}$ denote the complex interpolation functor. Then we can consider an operator $\tilde{A}$ in the base space $\tilde{X}=X^{\alpha}$ with the domain $D(\tilde{A})=\tilde{X}^{1}=X^{\alpha+1}$ as a restriction of the operator $A$, which means that $\tilde{A} u=A u$ for all $u \in X^{\alpha+1}$.

Using Sobolev's embedding theorem (see [1, Theorem 5.4, pp. 97-98]) we know that $W^{k, p}(\Omega) \subseteq C^{m+\mu}(\Omega)$ if and only if $0<\mu \leq k-m-\frac{n}{p}<1$. It implies that $k=m+1$. We will find the conditions which allow us to select $p \in[1, \infty)$ such that the solutions of the problem 4.1 will belong to the space $C^{m+\mu}(\Omega)$.

Taking $p \geq p_{0}=\frac{n}{-\mu+m-k}$ we have

$$
k-m-\frac{n}{p} \geq k-m-n \frac{-\mu+m-k}{n}=\mu,
$$

what shows, that $p_{0}$ is the smallest exponent which assures the embedding of the Sobolev space $W^{k, p_{0}}(\Omega)$ in the space of continuous functions.

In this example operator $A$ is the Laplace operator with Dirichlet boundary conditions, so to find continuous solutions of (4.1) we have to know that the base space is contained in $C^{\varepsilon}(\Omega)$ for some $\varepsilon \in(0,1)$, which is satisfied if the domain $D(A)$ is contained in $C^{2+\varepsilon}(\Omega)$. Taking the base space typically as 
$W_{0}^{1, p}(\Omega)$ and $D(A)=W_{B}^{3, p}(\Omega)$, we have to show that $W^{3, p}(\Omega) \subseteq C^{2+\varepsilon}(\Omega)$. This is true for $p \geq p_{0}=\frac{n}{1-\varepsilon}$.

It means that when we consider the problem (4.1) in the base space $W_{0}^{1, p}(\Omega)$ with $p \geq p_{0}$, then every solution $u$ has the property that $\Delta u$ is continuous on $\Omega \subseteq \mathbb{R}^{n}$ and so is $u_{t}$.

The theorem presented below gathers what was shown in the example.

TheOREM 4.2. Let $\Omega \subseteq \mathbb{R}^{n}$ be a bounded domain with $\partial \Omega \in C^{2}, p \in[1, \infty)$. Let us consider the Cauchy problem 4.1 in the base space $W_{0}^{1, p}(\Omega)$. Then, if $p \geq p_{0}=\frac{n}{1-\varepsilon}$, every solution $u \in W_{B}^{3, p}(\Omega)$ of 4.1 has the property that $u_{t}$ and $\Delta u$ are continuous on $\Omega \subseteq \mathbb{R}^{n}$.

EXAMPLE 4.3. Let us consider following problem

$$
\left\{\begin{array}{l}
u_{t}-\Delta u=0 \quad \text { in } D_{T}=[0, T] \times \Omega, \\
\left.u\right|_{\partial \Omega}=0, \\
u(0, x)=u_{0}(x), \quad x \in \Omega
\end{array}\right.
$$

where $T>0$ and $\Omega \subseteq \mathbb{R}^{n}$ is a bounded domain with $\partial \Omega \in C^{2}$. We will search for the solutions $u$ in the base space $W_{0}^{1, p}\left(D_{T}\right)$. Our aim is to set $p \in[1, \infty)$ such that all solutions are continuous, i.e. $W^{1, p}\left(D_{T}\right) \subseteq C^{\mu}\left(D_{T}\right)$ for some $\mu \in(0,1)$. Let us take $p \geq p_{0}=\frac{n+1}{1-\mu}$. Then we see that $1-\frac{n+1}{p} \geq$ $\geq 1-\frac{n+1}{p_{0}}=\mu>0$, and from Sobolev's embedding theorem we conclude that $W^{1, p}\left(D_{T}\right) \subseteq C^{\mu}\left(D_{T}\right)$.

Since $u \in W^{1, p}\left(D_{T}\right)$ we know that all derivatives $u_{t}, u_{x_{i}}$ for $i \in\{1, \ldots, n\}$ belong to the space $L^{p}\left(D_{T}\right)$ and using equations 4.2 we claim that $\Delta u=$ $u_{t} \in L^{p}\left(D_{T}\right)$.

Now, our purpose is to show that $u$ belongs to $W^{2, p}\left(D_{T}\right)$. Knowing that $u \in W_{0}^{1, p}(\Omega)$ and using the Calderón-Zygmund type estimations (see [5, Theorems 8.12 and 8.13 , pp.186-187]) we get that $u \in W^{2, p}(\Omega)$ and fulfills an estimate

$$
\|u\|_{W^{2, p}(\Omega)} \leq C\left(\|\Delta u\|_{L^{p}(\Omega)}+\|u\|_{L^{p}(\Omega)}\right)=C\left(\left\|u_{t}\right\|_{L^{p}(\Omega)}+\|u\|_{L^{p}(\Omega)}\right),
$$

for some constant $C>0$ depending on $\Omega, n$. Then, for every multiindex $\alpha$ with $|\alpha|=2$ we have $D^{\alpha} u \in L^{p}(\Omega)$, so there exist constants $M_{\alpha}$ such that $\left\|D^{\alpha} u\right\|_{L^{p}(\Omega)} \leq M_{\alpha}<\infty,(|\alpha|=2)$. We have

$$
\|u\|_{W^{2, p}\left(D_{T}\right)}=\sum_{|\alpha|=2}\left\|D^{\alpha} u\right\|_{L^{p}\left(D_{T}\right)}+\|u\|_{W^{1, p}\left(D_{T}\right)},
$$


thus we obtain the estimation:

$$
\begin{aligned}
\|u\|_{W^{2, p}\left(D_{T}\right)} & =\sum_{|\alpha|=2}\left(\int_{0}^{T} \int_{\Omega}\left|D^{\alpha} u\right|^{p} d x d t\right)^{1 / p}+\|u\|_{W^{1, p}\left(D_{T}\right)} \\
& \leq \sum_{|\alpha|=2}\left(\int_{0}^{T} M_{\alpha}^{p} d t\right)^{\frac{1}{p}}+\|u\|_{W^{1, p}\left(D_{T}\right)} \\
& =\sum_{|\alpha|=2} M_{\alpha} \cdot T^{\frac{1}{p}}+\|u\|_{W^{1, p}\left(D_{T}\right)}<\infty .
\end{aligned}
$$

This example showed that, under our assumptions and when $p>\frac{n+1}{1-\mu}$, every solution $u$ of 4.2 which belongs to $W_{0}^{1, p}\left(D_{T}\right)$, is also an element of the space $C^{\mu}\left(D_{T}\right) \cap W^{2, p}\left(D_{T}\right)$. Consequently, the above example lead us to the conclusion.

THEOREM 4.4. Let $\Omega \subseteq \mathbb{R}^{n}$ be a bounded domain with $\partial \Omega \in C^{2}, T>0$, and $p \in[1, \infty)$. Assume that $u \in W_{0}^{1, p}\left(D_{T}\right)$ is a solution of the problem (4.2). If $p>p_{0}=\frac{n+1}{1-\mu}$ then the function $u$ belongs to the space $C^{\mu}\left(D_{T}\right) \cap W^{2, p}\left(\bar{D}_{T}\right)$.

\section{References}

[1] Adams R.A., Sobolev spaces, Academic Press, New York-San Francisco-London, 1975.

[2] Cholewa J.W., Dłotko T., Global attractors in abstract parabolic problems, Cambridge University Press, Cambridge, 2000.

[3] Dawidowski Ł., Scales of Banach spaces, theory of interpolation and their applications, Wydawnictwo Uniwersytetu Śląskiego, Katowice, 2012.

[4] Evans L.C., Partial differential equations, Graduate Studies in Mathematics 19, American Mathematical Society, Providence, Rhode Island, 1998.

[5] Gilbarg D., Trudinger N., Elliptic partial differential equations of second order, Reprint of the 1998 edition, Springer-Verlag, Berlin, 2001.

[6] Henry D., Geometric theory of semilinear parabolic equations, Lecture Notes in Mathematics 840, Springer-Verlag, Berlin, 1981.

[7] Komatsu H., Fractional powers of operators, Pacific J. Math. 19 (1966), 285-346.

[8] Yagi A., Abstract parabolic evolution equations and their applications, Springer Monographs in Mathematics, Springer-Verlag, Berlin, 2010.

Institute of Mathematics

UNIVERSITY OF Silesia

BANKOWA 14

40-007 Katowice

POLAND

e-mail: lukasz.dawidowski@us.edu.pl 